Wilfrid Laurier University

Scholars Commons @ Laurier

Physics and Computer Science Faculty

Publications

Physics and Computer Science

$11-2009$

\title{
Miniature High-Sensitivity High-Temperature Fiber Sensor with a Dispersion Compensation Fiber-Based Interferometer
}

\author{
Bo Dong \\ Wilfrid Laurier University \\ Li Wei \\ Wilfrid Laurier University, Iwei@wlu.ca \\ Da-Peng Zhou \\ University of Waterloo
}

Follow this and additional works at: https://scholars.wlu.ca/phys_faculty

\section{Recommended Citation}

Dong, Bo; Wei, Li; and Zhou, Da-Peng, "Miniature High-Sensitivity High-Temperature Fiber Sensor with a Dispersion Compensation Fiber-Based Interferometer" (2009). Physics and Computer Science Faculty Publications. 7.

https://scholars.wlu.ca/phys_faculty/7

This Article is brought to you for free and open access by the Physics and Computer Science at Scholars Commons @ Laurier. It has been accepted for inclusion in Physics and Computer Science Faculty Publications by an authorized administrator of Scholars Commons @ Laurier. For more information, please contact scholarscommons@wlu.ca. 


\title{
Miniature high-sensitivity high-temperature fiber sensor with a dispersion compensation fiber-based interferometer
}

\author{
Bo Dong, ${ }^{1, *}$ Li Wei $^{1,2}$ and Da-Peng Zhou ${ }^{2}$ \\ ${ }^{1}$ Department of Physics and Computer Science, Wilfrid Laurier University, Waterloo, \\ Ontario N2L 3C5, Canada \\ ${ }^{2}$ Department of Physics and Astronomy, Guelph-Waterloo Physics Institute, University of Waterloo, \\ Waterloo, Ontario N2L 3G1, Canada \\ ${ }^{*}$ Corresponding author: dbo1978@163.com
}

Received 13 August 2009; revised 25 October 2009; accepted 30 October 2009; posted 2 November 2009 (Doc. ID 115664); published 12 November 2009

\begin{abstract}
A miniature high-sensitivity, high-temperature fiber sensor with an interferometer based on a bare small-core-diameter dispersion compensation fiber (DCF) is reported. The sensing head is a singlemode-fiber (SMF) DCF configuration formed by a $4 \mathrm{~mm}$ long bare DCF with one end connected to the SMF by a fusion splicing technique and the other end cleaved. Due to the large mode index difference and high thermo-optic coefficient induced by two dominative interference modes, a miniature hightemperature fiber sensor with a high sensitivity of $68.6 \mathrm{pm} /{ }^{\circ} \mathrm{C}$ is obtained by monitoring the wavelength shift of the interference spectrum. This type of sensor has the features of small size, high sensitivity, high stability, simple structure, and low cost. () 2009 Optical Society of America
\end{abstract}

OCIS codes: $\quad 060.2310,060.2370$.

\section{Introduction}

To meet the increasing demands of modern industry, different types of fiber temperature sensor based on fiber gratings, High-birefringence (Hi-Bi) fiber interferometers, multimode fiber (MMF) interferometer, etc., have been developed. Among them, the use of fiber Bragg gratings [1-3] is particularly attractive for their small size, wavelength encoding, and wavelength multiplexing features, but the temperature sensitivity of fiber Bragg gratings is limited $\left(\sim 10 \mathrm{pm} /{ }^{\circ} \mathrm{C}\right)$. The use of long-period gratings [4] provides a high sensitivity to temperature, but longperiod gratings easily suffer from the bend effect. Moreover, the fabrication technique for fiber Bragg gratings and long-period gratings is complicated, which results in an expensive cost. Hi-Bi fiber interferometer temperature sensors [5] have the advan-

0003-6935/09/336466-04\$15.00/0

(C) 2009 Optical Society of America tages of easy fabrication and high sensitivity, but they need long Hi-Bi fibers and polarization controller or polarizers, which is impractical for distributed sensing. Recently MMF interference temperature sensors have attracted much attention for their advantages of low-cost and high-temperature operation. A conventional type of sensor [6] using stepindex MMF with a large core diameter has low temperature sensitivity $\left(\sim 15 \mathrm{pm} /{ }^{\circ} \mathrm{C}\right)$ and needs a long section of MMF, about $50 \mathrm{~mm}$; although the temperature sensitivity was improved to $58.5 \mathrm{pm} /{ }^{\circ} \mathrm{C}$ by using a large-core-diameter graded-index MMF [7], that required a $1.8 \mathrm{~m}$ long graded-index $\mathrm{MMF}$, and high-temperature operation was not demonstrated. The above reported sensors with long MMFs are not practical for real application for two main reasons. First, the long MMF is easily influenced by ambient disturbances; second, for high-temperature application, the above sensing head has to adopt the bare fiber in order to avoid fiber coating burning, which induces undesirable strain to the sensing 
head. Obviously, it is difficult to fabricate a bare fiber sensing head by using a long section of MMF.

Miniature fiber sensors are highly desirable in high-temperature sensing. Although miniature high-temperature fiber sensors based on FabryPerot (FP) interference [8-10] were demonstrated, they needed complicated fabrication process such as etching [8] and laser micromachining [9] to form a single $\mathrm{FP}^{-}$cavity, or cascading three sections of fibers [a photonic crystal fiber, a hollow optical fiber and a single-mode fiber (SMF)] to form a hybrid FP cavity [10], and the length of the FP sensors constructed with fiber is generally more than $1 \mathrm{~cm}$. On the other hand, a miniature high-temperature fiber sensor based on modal interference was fabricated with a $1.2 \mathrm{~cm}$ length by cascading two MMFs and three SMFs [11]; however, the structure of the sensing head is not simple, and the fabrication processes is not easy.

In this paper, we present a miniature highsensitivity, high-temperature fiber sensor with a bare small-core dispersion compensation fiber (DCF)-based interferometer. Within the $\mathrm{C}$ band, the DCF can act as an MMF. The sensing head has an SMF-DCF configuration formed with a $4 \mathrm{~mm}$ long bare DCF with one end being spliced to a section of SMF and the other end being cleaved. To the best of our knowledge, the proposed $4 \mathrm{~mm}$ long fiber sensor is the shortest fiber-based sensor to this day. Within the temperature measurement range of $25^{\circ} \mathrm{C}-600{ }^{\circ} \mathrm{C}$, a high sensitivity of $68.6 \mathrm{pm} /{ }^{\circ} \mathrm{C}$ is obtained by monitoring the wavelength shift of the interference spectrum. Moreover, our proposed sensor can be made 22 or 84 times smaller than the two sensors in $[6,7]$, considering that all the sensors have the same free spectral range (FSR). This is because the small-core-diameter DCF has a much larger mode index difference between the two interference modes than does the conventional step-index or gradedindex MMF. Such a unique property allows us to build ultracompact sensing heads, which are more suitable for distributed sensing, as well as biomedical or chemical sensing applications. This type of sensor has the features of small size, high sensitivity, high stability, simple structure, and low cost.

\section{Experimental Setup and Principle}

Figure 1 shows the schematic diagram of the experimental ${ }^{-}$setup. The temperature is controlled by a tube furnace (Thermolyne type 21100). The temperature of the fiber sensor was measured by a type- $K$ thermocouple, which was connected to a digital thermometer. The sensor head was attached to the thermocouple, and both of them were inserted into the tube furnace. A C-band broadband source (BBS) with an amplified spontaneous emission spectrum centered at $1550 \mathrm{~nm}$ was used as the light source. The interference spectra were monitored by an optical spectrum analyzer (OSA, Agilent 86142B).

The sensing head, as shown in the inset of Fig. 1, is fabricated by connecting a $4 \mathrm{~mm}$ long bare DCF to a

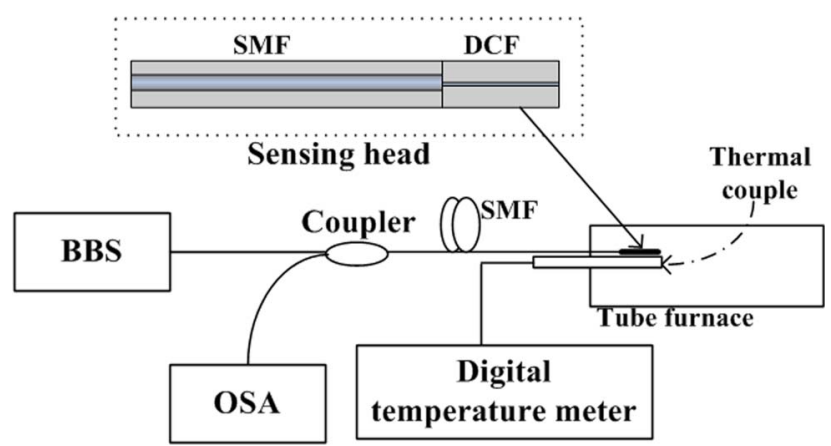

Fig. 1. (Color online) Schematic diagram of the experimental setup (inset, structure of the sensing head).

standard communications SMF, which serves as an interferometer based on intermode interference. The DCF has a core/cladding diameter of 1.9/ $115.7 \mu \mathrm{m}$, a large dispersion parameter of $-270 \mathrm{ps} /$ $\mathrm{nm} / \mathrm{km}$ at $1550 \mathrm{~nm}$, and a cutoff wavelength of $1663 \mathrm{~nm}$. Within the $\mathrm{C}$ band, the DCF can act as an MMF. The free end of the DCF was cleaved as a mirror to reflect the light back to the DCF. The emitted light of the BBS propagates to the end surface of the sensing head through a $3 \mathrm{~dB}$ coupler, and then the reflected light propagates to the OSA through the coupler again. The fundamental mode that propagates along the SMF will couple into the DCF and excite different interference modes at the SMF-DCF splice point. Reflected back to the DCF by the cleaved end surface of the DCF, these modes will interfere and recouple into the SMF at the DCFSMF splice point.

Figure 2 shows the measured reflectivity spectrum of the interferometer and the transmission power spectrum of the applied BBS. The interference can be approximately considered to be produced by two dominant modes, which indicates that other weak modes carry few powers and slightly modulate the interference pattern. The phase difference between the two dominant modes after propagating through the DCF can be written as $\varphi_{m}=4 \pi B L / \lambda$, where

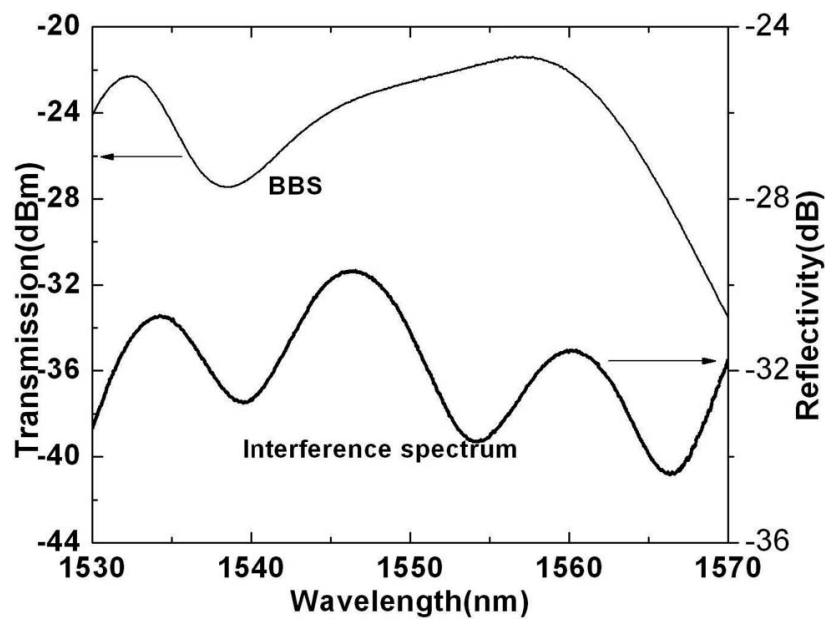

Fig. 2. Interference spectrum of the DCF interferometer. 
$B=n_{1}-n_{2}$ is the mode index difference of the two dominant modes, and $n_{1}, n_{2}$ are the mode indices of the two modes respectively; $\lambda$ is the signal wavelength in vacuum and $L$ is the length of the DCF. The interference condition of the two dominant modes can be given by

$$
\varphi_{m}=\frac{4 \pi B L}{\lambda}=N 2 \pi \quad(N \text { is an integer })
$$

When ambient temperature changes, the mode index difference $B$ and the fiber length $L$ will change accordingly, and thus the interference spectrum will have a wavelength shift $\Delta \lambda$. According to Eq. (1) the relative wavelength shift $\Delta \lambda / \lambda$ caused by a temperature change $\Delta T$ can be expressed as

$$
\frac{\Delta \lambda}{\lambda} \approx(\alpha+\xi) \Delta T
$$

where $\alpha=d L / L d T$ is the thermal expansion coefficient of the DCF material, and $\xi=d B / B d T$ is the thermo-optic coefficient induced by the two interference modes in the DCF material. The FSR of the interfering spectrum can be approximately given by

$$
\mathrm{FSR}=\frac{\lambda^{2}}{2 B L}
$$

It can be seen that the FSR is inversely proportional to both the mode index difference $B$ and the length of the DCF L. According to Eq. (3) and Fig. 2, the mode index difference of our proposed DCF interference sensor is about $2.1 \times 10^{-2}$. However, the mode index difference of the high-temperature $105 / 125 \mu \mathrm{m} \mathrm{MMF}$ interference sensor [6] is about $9.6 \times 10^{-14}$, and that of the $100 / 140 \mu \mathrm{m} \overline{\mathrm{M} M F}$ temperature sensor [7] is only about $2.5 \times 10^{-4}$. The mode index difference of our proposed interference sensor is about 22 and 84 times larger than that of the two temperature sensors in $[6,7]$. In other words, our proposed sensor can be made by 22 or 84 times smaller than the two sensors above, considering that all the sensors have the same FSR. On the other hand, sensor resolution is a very important parameter and is dependent on wavelength measurement errors that are determined by the resolution of OSA and the bandwidth of the resonance peak. For a fixed value of $L$, a large value of $B$ gives rise to a small FSR and consequently a small bandwidth of the resonance peak, which will be beneficial to the sensor resolution. Therefore, the proposed $1.9 / 115.7 \mu \mathrm{m}$ DCF interference sensor with a large mode index difference can be miniaturized readily with a high-temperature resolution. The parameters of the refractive indices of the core and the cladding of the DCF are not known for us. The larger mode index difference of the DCF interferometer is attributed to the special design of the DCF.

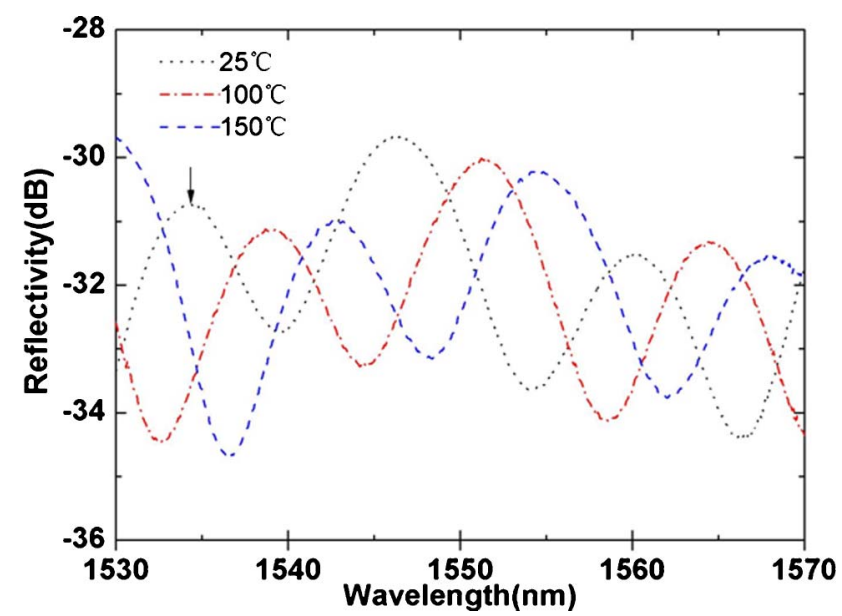

Fig. 3. (Color online) Wavelength shifts of the interference spectrum at different temperatures.

\section{Experimental Results and Discussion}

Since the BBS we used is not polarized, the transmission spectrum of the interferometer is polarization independent. The OSA with a resolution of $0.06 \mathrm{~nm}$ is used to measure the transmission spectrum of the interferometer. When the temperature increases, the DCF interference spectrum has a red shift while the spectrum almost keeps the same pattern, as shown in Fig. 3 . To monitor a broad wavelength shift, we measure the wavelength variation of the point at the arrow shown in Fig. 3, starting with the wavelength of $1534.28 \mathrm{~nm}$ at a room temperature of $25^{\circ} \mathrm{C}$. Figure 4 shows the measured relationship between the wavelength and the temperature. It can be seen that there is a good linear relationship between the wavelength and temperature, and the correlation coefficient square of $R^{2}$ reaches 0.9983 . Within the temperature range of $25^{\circ} \mathrm{C}-600^{\circ} \mathrm{C}$, the temperature sensitivity reaches $68.6 \mathrm{pm} /{ }^{\circ} \mathrm{C}$. Compared with the high-temperature MMF-based sensors [6], the proposed high-temperature sensor has higher sensitivity, which is mainly because of the higher

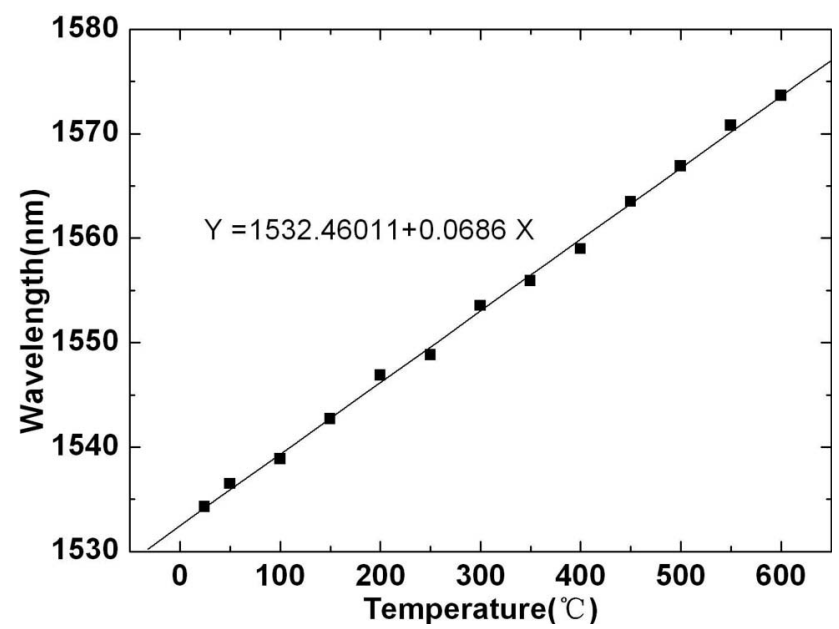

Fig. 4. Measured relationship between the wavelength and the temperature. 
thermo-optic coefficient. The thermal expansion coefficient is generally lower by 2 orders of magnitude than the thermo-optic coefficient for the optical fiber; ignoring the thermal expansion coefficient, the thermo-optic coefficient difference of the DCF used in our experiment is about $4.4 \times 10^{-5}$, while that of the MMF in [6] is about $9.7 \times 10^{-6}$. As the FSR of the proposed fiber sensor is much smaller than the bandwidth of the available BBS, the length of the sensing head can be shortened further to obtain ultracompact sensing heads. To test the influence of the ambient disturbance on the sensor, we sway the sensing head under several fixed temperatures, and we find that the interference spectrum does not change and has high stability under a fixed temperature, which is attributed to the small size of the sensing head. In addition, it can be seen that a cleaved surface used as a reflection mirror provides low reflection. However, the low reflection does not affect our measurement accuracy, since we measure the wavelength shift of the spectrum. The reflectivity can be increased by coating the DCF end surface with aluminum.

\section{Conclusions}

A miniature high-temperature fiber sensor with a bare small-core-diameter DCF-based interferometer has been proposed and demonstrated experimentally. Experimental results show that the DCF interference temperature sensor with an only $4 \mathrm{~mm}$ long bare DCF can be operated with high sensitivity of $68.6 \mathrm{pm} /{ }^{\circ} \mathrm{C}$ within the temperature measurement range of $25^{\circ} \mathrm{C}-600^{\circ} \mathrm{C}$. This type of sensor has the features of small size, high sensitivity, high stability, simple structure, and low cost. It is expected to be applied in smart and distributed fiber temperature sensing.

This work is supported by the Natural Science and Engineering Research Council of Canada (NSERC).
The authors acknowledge Sumitomo Electric Industries, Ltd. for providing the $1.9 \mu \mathrm{m} / 115.7 \mu \mathrm{m}$ DCF.

\section{References}

1. K. P. Koo and A. D. Kersey, "Bragg grating-based laser sensors systems with interferometric interrogation and wavelength division multiplexing," J. Lightwave Technol. 13, 1243-1249 (1995).

2. G. A. Ball, W. W. Morey, and P. K. Cheo, "Single- and multipoint fiber-laser sensors," IEEE Photon. Technol. Lett. 5, 267-270 (1993).

3. V. Bhatia and A. M. Vengsarkar, "Optical fiber long-period grating sensors," Opt. Lett. 21, 692-694 (1996).

4. Y. Liu, B. Liu, X. Feng, W. Zhang, G. Zhou, S. Yuan, G. Kai, and $\mathrm{X}$. Dong, "High-birefringence fiber loop mirrors and their applications as sensors," Appl. Opt. 44, 2382-2390 (2005).

5. A. N. Starodumov, L. A. Zenteno, D. Monzon, and E. De L. Rosa, "Fiber Sagnac interferometer temperature sensor," Appl. Phys. Lett. 70, 19-21 (1997).

6. E. Li, X. Wang, and C. Zhang, "Fiber-optic temperature sensor based on interference of selective higher-order modes," Appl. Phys. Lett. 89, 091119 (2006).

7. Y. Liu and L. Wei, "Low-cost high-sensitivity strain and temperature sensing using graded-index multimode fibers," Appl. Opt. 46, 2516-2519 (2007).

8. X. Chen, F. Shen, Z. Wang, Z. Huang, and A. Wang, "Micro-airgap based intrinsic Fabry-Perot interferometric fiber-optic sensor," Appl. Opt. 45, 7760-7766 (2006).

9. T. Wei, Y. Han, H. Tsai, and H. Xiao, "Miniaturized fiber inline Fabry-Perot interferometer fabricated with a femtosecond laser," Opt. Lett. 33, 536-538 (2008).

10. H. Y. Choi, K. S. Park, S. J. Park, U. Paek, B. H. Lee, and E. S. Choi, "Miniature fiber-optic high temperature sensor based on a hybrid structured Fabry-Perot interferometer," Opt. Lett. 33, 2455-2457 (2008).

11. L. V. Nguyen, D. Hwang, S. Moon, D. S. Moon, and Y. Chung, "High temperature fiber sensor with high sensitivity based on core diameter mismatch," Opt. Express 16, 11369-11375 (2008). 